\title{
Russische Zeitschrift für Haut- und Geschlechtskrankheiten.
}

\author{
Juli 1912.
} pag. 3.

Hogrow. Mikrosporie und ihre Bekämpfung.

Im Lehrjahr 1911-12 kamen in der Moskauer Klinik ambulatorisch $129^{\prime}$ Fälle parasitärer Hauterkrankungen zır systematischen Untersuchung, 26 Fälle von Favus, 81 Fälle von Herpes tonsurans, 2 Fälle von Kerion, 20 Fälle von Mikrosporie; unter diesen letzteren 11 Patienten, die aus einer kleinen Schulepidemie stammen und 3 aus Tula. Aus den Schlußsätzen der Arbeit sei hervorgehoben:

Die untersuchten Fälle von Mikrosporie sind tierischen Ursprungs (M. lanosum). Zu ihrer Bekämpfung sowie der anderen parasitären Haaraffektionen ist die Untersuchung der Schulkinder bei ihrer Aufnahme und ihre periodische Untersuchung im Laufe des Schuljahrs durch dermatologisch geschulte Ärzte notwendig. Die erkrankten Kinder bedürfen einer sorgfältigen Überwachung, Isolierung und energischen Behandlung. Die entsprechend dem gegenwärtigen Stand der Wissenschaft zu fordernde Röntgenbehandlung wird derzeit (unter den öffentlichen Instituten Moskaus) nur an der Hautklinik geübt. Für die exakte Diagnose der Erkrankung und die Bestimmung der endgültigen Heilung ist die mikroskopische und mikrobiologische Untersuchung der Haare unerläBlich. Mit Rücksicht auf die stete Zunahme der parasitären Haarerkrankungen sind im Zentrum größerer Schuldistrikte Einrichtungen zur Behandlung der Haaraffektionen zu treffen. Zwei Photogramme erkrankter Haare, aus denen die Verteilung ron Sporen und Mykel hervorgeht, sind der Arbeit beigefügt.

Troitzka, A. Zur Leprabehandlung mittels Salvarsan. pag. 10 .

Im Augustheft 1911 dieser Zeitschrift wurde über eine Patientin berichtet, die nach $(0 \cdot 4,0 \cdot 5,0 \cdot 6,0 \cdot 7)$ Salvarsan Besserung der makulo-tuberösen Form der Lepra zeigte. Die Behandlung mußte infolge einer Phlegmone der rechten Hand unterbrochen werden. Vom 8. Oktober bis 26 . November wurden 4 intravenöse Salvarsaninjektionen $(0.6,0.7,0.75,0.8)$ appliziert. Nach dem dritten, besondel's aber wach dem vierten Einlauf kam es zu schweren Intoxikationserscheinungen, die bis $8 \mathrm{bzw}$. 14 Tage lang anhielten. Unter dem Einfluß dieser Injektionen ging die Rückbildung der Infiltrate in langsamerem Tempo vor sich, als unter der der ersten Serie. 
Nach 6wöchentlicher Unterbrechung. wurden wieder fünf Injektionen à 0.6 und eine Injektion Neosalvarsan 0.9 gemacht. Es konnte ein Wachstum der Augenbrauen (inneres Drittel) konstatiert werden, während die $\mathrm{Hansenschen} \mathrm{Stäbchen} \mathrm{sich}$ an Zahl nicht verringerten, die Sensibilität sich nicht veränderte. An den Knoten und Infiltraten zeigte sich eine wesentliche Besserung. Doch konnte ein Neuauftreten derselben nicht verhindert werden, was vielleicht der Verringerung der Dosis auf 0.6 zuzuschreiben ist.

Mit Rücksicht auf den anfänglich günstigen Effekt wird die Frage der Möglichkeit der Gewöhnung an Salvarsan diskutiert und die Fortsetzung der Versuche an einem großen Material mit entsprechenden Unterbrechungen empfohlen.

Abulow. Betrachtungen über die Wirkung des Salvarsans bei Syphilis und einigen anderen Erkrankungen. 342 Fälle. pag. 21.

Die Schlußfolgerungen des Autors: Die Therapia magna sterilisans wird bei den ersten Entwicklungsstadien der Syphilis durch eine Injektion nicht erreicht. In der Frühperiode (Ulcus durum) kann Salvarsan die Erscheinungen der S. entweder vollständig verhindern oder lange hinausschieben. Es bildet aber ein mächtiges Hilfsmittel bei der Behandlung der verschiedenen Symptome. Bei parasyphilitischen Erkrankungen bringt $\mathrm{S}$. in vielen Fällen symptomatische Besserung hervor, wobei Atrophia n. optici keine Kontraindikation für seine Anwendung bildet. Sowohl subkutane, intramuskuläre, als auch intravenöse Injektionen werden in der ersten Hälfte der Schwangerschaft gut vertragen. Auf die Schwangeren und die Frucht ist es von günstigem Einfluß, indem es den Abort verhindert. Dem Aussehen nach scheinen die Kinder gesund. Die Behandlung. der Säıglinge durch Salvarsaneinspritzungen der stillenden Mütter ist größtenteils erfolglos. Weder die wiederholte S. noch die kombinierte Hg-S.-Behandlung verhindert das Auftreten von Rezidiven. Bei Psoriasis vulgaris und Sycosis parasitaria lassen sich keine Erfolge erzielen. Bei Malaria erweist es sich in allen Fällen wirksam, doch nur für kurze Dauer. Tuberkulose und Nephritis bilden keine Gegenanzeige. Meschtscherski. Hôpital International, das Institut von B. Motz in Paris. pag. 90.

Beschreibung der urologischen Privatklinik, der daselbst gebräuchlichen Untersuchungs- und Behandlungsmethoden und Würdigung derselben als Lehranstalt für ein internationales Ärztepublikum.

Richard Fis chel (Bad Hall). 